\title{
Tracking Methods for Medical Augmented Reality
}

\author{
Abhilash Pandya, M.S. ${ }^{1}$, Mohamad Siadat, M.S. ${ }^{1}$, Lucia Zamorano, M.D. Dr. \\ Med. ${ }^{1}$, Jainxing Gong, Ph.D. ${ }^{1}$, Qinghang Li. ${ }^{1}$, M.D. Ph.D, James Maida, M.S. ${ }^{2}$, \\ Ioannis Kakadiaris, Ph. $\mathrm{D}^{3}$
}

1. Neurosurgery Department suite 930, 4160 John R, Detroit, Mi. 48201

2. NASA, Johnson Space Center, Houston, Tx.

3. University of Houston, Houston, Tx.

This work is partially funded by NASA grant 99-HEDS-01-079

apandya@neurosurgery.wayne.edu

\section{Introduction}

Recently, the capabilities of real-time PC-based video image processing and computer graphic systems converged to make possible the display of a virtual graphical image correctly registered with a view of the 3D environment of the user's object of interest. The generation of an Augment Reality scene can now be done with a PC computer graphics system. An Augmented Reality (AR) system generates a composite view for the user. It is a combination of the real scene viewed by the user and a virtual scene generated by the computer (a 3D model) that augments the scene with additional information. Fig. 1 displays one possible AR scene where a phantom is overlaid with graphics models of tumors. One of the most important issues to consider for a very accurate AR application is the method for tracking the various elements of the environment such as the video camera.

\section{Methods}

For the camera tracking there are at least three different methods: 1) tracking using an infrared camera tracking system, 2) tracking using a precise robot arm and 3) a camera calibration method using pattern recognition techniques. Note that for the two former methods we need to estimate the camera parameters (calibrate the camera) to generate an accurate AR scene. An infrared stereo-camera with its LEDs is considered enough hardware for both camera calibration and camera tracking. Tracking can be achieved by a robot in which the geometrical information (position and orientation of the mounted camera) is calculated through forward kinematics. A robot also provides enough information for the camera calibration procedure. The third method for camera tracking uses the captured frames in which several well-known patterns are tracked. This method works based on image processing techniques [1] and needs minimum hardware requirements. In the third method the information needed for camera calibration procedure [2] has to be provided either by a special geometrical instrument or through the two former methods. So the camera calibration method is used for camera tracking as well as camera parameter estimation (camera calibration). 


\section{Results/Conclusions}

There are some limitations and strengths for using each of the systems outlined for camera tracking. Line-of-site and lighting condition issues exist for both the pattern recognition and infrared tracking. The virtual objects will only appear when the tracking marks are in view and the lighting conditions are properly adjusted. A robotics-based camera overcomes both of these problems. There are also range issues. For the pattern recognition system, the larger the physical pattern the further away the pattern can be detected and so the greater the tracking volume. The infrared camera distance to the phantom limits the IR tracking (typically the range is 1 meter). The robotic solution is dependent on the robotic kinematics and the range of motion of each of the joints. For the restricted volume needed for neurosurgery applications, all the mentioned methods could be potentially used [4,5]. For redundancy, a solution that relies on more than one modality would be advantageous. For instance, using infrared guided tracking with image processing would provide continuous tracking during non-optimal scenarios. This paper represents work in progress.

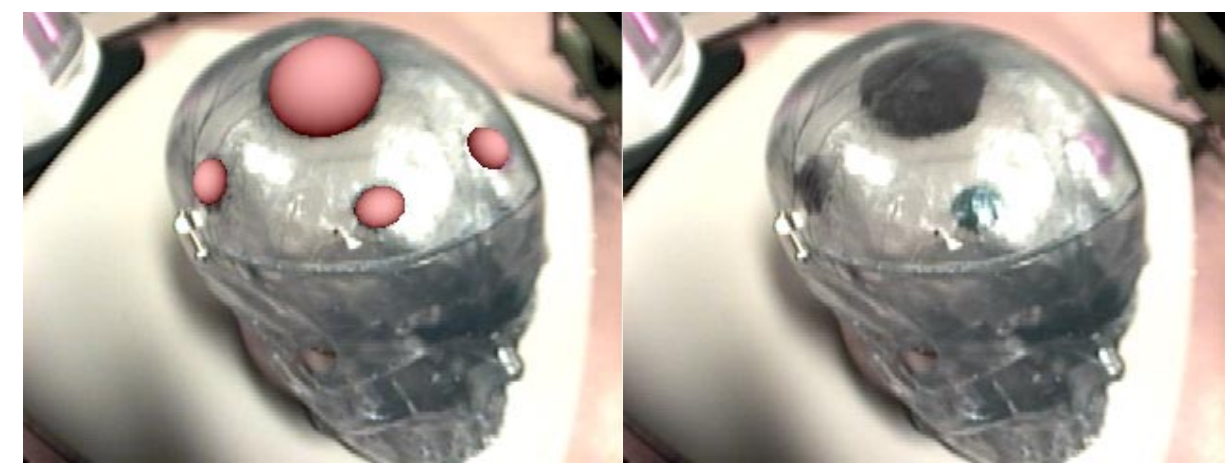

Fig. 1. An AR scene: A see-through phantom is overlaid with graphics models of the "tumors".

\section{References}

1. ArtoolKit http://www.hitl.washington.edu/resarch/shared_space/download/

2. Weng J., Cohen P., Herniou M., "Camera Calibration with Distortion Model and Accuracy Evaluation,” IEEE Trans. PAMI, vol. 14, no. 10, 1992.

3. Pandya A., Siadat M., Gong J., Li Q, Zamorano L., Maida J., "Towards Using Augmented Reality for Neurosurgery”, Medicine Meets Virtual Reality, Jan 2001.

4. Pandya A., Zamorano L., Siadat M., Gong J., Li. Q, Maida J., "Advanced Surgical Image Environments", The $5^{\text {th }}$ Annual Neurosurgery Detroit Symposium-Neurosurgery in the $21^{\text {st }}$ Century, Nov. 3-5th 2000 